\title{
Metodologias, ferramentas e aplicações da Inteligência Artificial nas diferentes linhas do combate a Covid-19
}

\section{Bárbara Coelho Neves}

Pós-Doutora em Ciência da Informação, pela Universidade de Brasília (UnB). Doutora em Educação e Mestra em Ciência da Informação, pela Universidade Federal da Bahia (UFBA). Professora Adjunta do Instituto de Ciência da Informação da UFBA e Docente Permanente do Programa de PósGraduação em Ciência da Informação da UFBA. Pesquisadora líder do LTI Digital.

barbaran@ufba.br

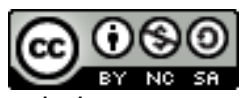

Este trabalho está licenciado com uma Licença Creative Commons - AtribuiçãoNãoComercial-Compartilhalgual 4.0

Internacional.

\section{Resumo}

O tema deste artigo trata da inteligência artificial (IA) no contexto da informação em saúde. Tem como objetivo principal apresentar um conjunto de metodologias, ferramentas e aplicações da inteligência artificial nas diferentes linhas do combate ao novo coronavírus e a Covid-19. Especificamente, exemplifica tecnologias de IA (dados tabulares, processamento de linguagem natural e visão computacional), utilizadas durante a pandemia nos campos da saúde e destaca as principais aplicações da IA no combate a Covid-19. Quanto à metodologia, foi um estudo com abordagem qualitativa, descritivo e de levantamento sistemático com apoio de revisão de literatura. Como principal resultado apresenta os avanços da transformação digital que a sociedade está passando, principalmente, nos campos que envolvem as ações de mitigação do novo coronavírus. Considera finalmente que o movimento circular entre tecnologias, inovação e comportamento se retroalimentam na sociedade e a inteligência artificial perpassa de forma transversal este processo. Talvez isso seja ainda mais forte e visível de ser observado durante a pandemia da Covid- 19 .

Palavras-chave: Inteligência Artificial. Transformação Digital. Covid-19. Novo coronavírus. SARS-Cov-2. Pandemia de Covid-19. Informação em Saúde.

\section{Methodologies, tools and applications of artificial} intelligence in the different lines of the fight against (Covid-

\section{Abstract}

The theme of this article deals with artificial intelligence (AI) in the context of health information. Its main objective is to present a set of methodologies, tools and applications of artificial intelligence in the different lines of the fight against new coronavirus (Covid-19). Specifically, it exemplifies Al technologies (tabular data, natural language processing and computer vision), used during the pandemic in the health fields and highlights the main applications of Al in combating Covid-19. As for the methodology, it was a study with a qualitative, descriptive and systematic field approach supported by literature review. As a main result it presents the advances of digital transformation that society is going through, mainly, in the fields that involve coronavirus mitigation actions. Finally, he considers that the circular movement between technologies, innovation and behavior feed back into society and artificial intelligence cuts across this process. Perhaps this is even stronger and more visible to be observed during this Covid19 pandemic.

Keywords: Artificial intelligence. Digital Transformation. Covid-19. New coronavirus. SARS-Cov-2. Covid-19 pandemic. Health Information. 


\section{Introdução}

As pandemias sempre aconteceram na humanidade. Até o presente, foram registradas a varíola (1.145 a.C. a 1977), a peste bubônica (1333 a 1351), a cólera (1817 a 1824), a tuberculose (1850 a 1950), a gripe espanhola (1917 a 1919) e a H1N1 (2009 a 2010). O coronavírus (SARS-Cov-2), causador da Covid-19, apresentou seus primeiros casos em final de 2019 e se caracteriza como a primeira pandemia num mundo em processo de transformação digital e conectado por inteligência artificial (IA). A saúde concerne diretamente à preservação da vida e ao desenvolvimento das capacidades humanas, sendo uma necessidade humana básica em sentido restrito (LIMA; FINAMOR, 2019).

A tecnologia é um aliado no protagonismo de médicos e das políticas de saúde no combate aos vírus e um dos seus principais elementos de apoio é a comunicação da informação de maneira rápida. Atualmente a sociedade possui um conjunto de tecnologias disruptivas que possui um maestro: a IA. Essas tecnologias convergem, principalmente, no campo da saúde interessadas em combater um inimigo comum, tornando a maneira de lidar com este vírus, diferente da abordagem com outros agentes causadores de doenças pandêmicas do passado.

O debate inicial que se inaugura neste artigo se sustenta na relevância de propor aproximações entre cientistas da informação e os temas da IA, que nesse contexto torna-se possível no eixo da informação em saúde. Os estudos do vasto e complexo campo da informação em saúde tem revelado "[...] que não existe uma parceria efetiva entre bibliotecários e outros profissionais de saúde, pois se faz necessária à interação e integração entre as atividades de cada um em busca do melhor atendimento às necessidades informacionais e demandas de saúde" (LIMA; FINAMOR, 2019, p.129).

Sistemas computadorizados de apoio à decisão clínica têm indicado um alto grau de acurácia em suas propostas diagnósticas. Ao mesmo tempo, a computação cognitiva da IBM, Watson, registrou toda a informação médica disponível na PubMed e Medline, tornando mais fácil, idealmente, o acesso à informação em saúde (LOBO, 2018).

Diante disso, este artigo visa apresentar um conjunto de metodologias, ferramentas e aplicações da IA que estão sendo aplicadas nas diferentes linhas do combate ao novo coronavírus. Para isso, tem como objetivos específicos exemplificar as tecnologias de IA (dados tabulares, processamento de linguagem natural e visão computacional) utilizadas durante a pandemia nos campos da saúde, e busca destacar as principais aplicações da IA no combate a Covid-19.

Quanto aos materiais e métodos utilizados para elaboração do estudo, foi utilizada abordagem qualitativa, pois as análises foram feitas a partir do próprio pesquisador (LAVILLE; DIONE, 1999), em estudo do tipo descritivo, visando se juntar a outros que pretendem esclarecer a relação da IA com a pandemia Covid-19. A metodologia tem características de levantamento, com apoio de pesquisa documental e da revisão de literatura.

Este artigo está dividido em duas partes. A primeira aborda conceitos ligados à inteligência artificial e as metodologias usadas após o fato de reconhecimento de que a Covid-19 se trata de uma pandemia. A segunda parte aborda as principais aplicações da IA para combater o vírus, com a ilustração de exemplos e onde a IA pode atuar após a problemática da pandemia, se constituindo assim como legado dos avanços impulsionados pelo evento.

\section{Metodologias da inteligência artificial utilizadas durante a pandemia}

Embora a IA esteja a cada dia mais ativa no campo da informação em saúde, percebe-se que a Biblioteconomia ainda não consolidou de fato uma aproximação efetiva com os seus campos. A IA é um ramo da ciência da computação que se propõe a desenvolver sistemas que simulem a capacidade humana na percepção de um problema, identificando seus componentes e, com isso, resolver problemas e propor/tomar decisões (LOBO, 2018).

É perceptível os fortes indícios de que as inovações disruptivas no eixo da informação em saúde estão muito mais presentes em nosso contexto nacional do que imaginamos, e seu potencial crescimento exponencial não permite que os profissionais da área da Ciência da Informação esmoreçam frente aos seus avanços. Faz-se necessária a sensibilização de pesquisadores, gestores, arquivistas, bibliotecários, sanitaristas e quaisquer outros atores que trafegam à luz dos dados e informação em 
saúde, para que seja considerado esse novo movimento em suas premissas, pesquisas e práticas no contexto da saúde (LEAL NETO; ALBUQUERQUE; SOUZA; CESSE; CRUZ, 2017).

A IA é um grande guarda-chuva que abriga uma série de tecnologias distintas que podem ser usadas de forma isolada ou em conjunto para resolução de uma determinada demanda. Suas ferramentas compreendem: análise de dados tabulares, processamento de imagem, visão computacional, dentre outras.

Considerando que se falará a seguir sobre metodologias, esse texto compreende que o conceito de inteligência artificial abriga machine learning que, por sua vez, tem como capacidade mais especifica a deep learning. A seguir apresentamos cada uma dessas tecnologias com a intenção de entendermos cada uma delas no combate ao vírus.

\subsection{Dados tabulares por IA}

O uso das técnicas apresentadas no ensaio de Fernandes e Chiavegatto Filho (2018, p.15), insere-se no atual contexto de divulgação de "[...] dados abertos e de amplos volumes, por setores governamentais e privados, e da necessidade de integração e análise de dados de diferentes fontes e tipos: dados tabulares, dados textuais provenientes de redes sociais, dados audiovisuais e outros".

A inteligência artificial nos campos da saúde pode trabalhar associada a tabulação de dados em mineração (data mining) ou mineração textos (text mining). O uso de data mining, combinado com algoritmos de machine learning, "[...] pode auxiliar o especialista da saúde em momentos críticos que demandem decisões rápidas quando há uma deficiência dos recursos apresentados, por exemplo imagens de baixa resolução" (FERNANDES; CHIAVEGATTO FILHO, 2018, p.18).

A partir do momento que o novo coronavírus começou a se espalhar pelo globo, algumas iniciativas ganharam notoriedade apresentando dados que pudessem ser utilizados por algoritmos de IA. Um exemplo é o Hospital Johns Hopkins que desenvolveu um Centro de Recursos do Coronavírus para coletar e apresentar dados atualizados da pandemia pelo mundo para que possam ser processados por outras soluções com o uso de IA.

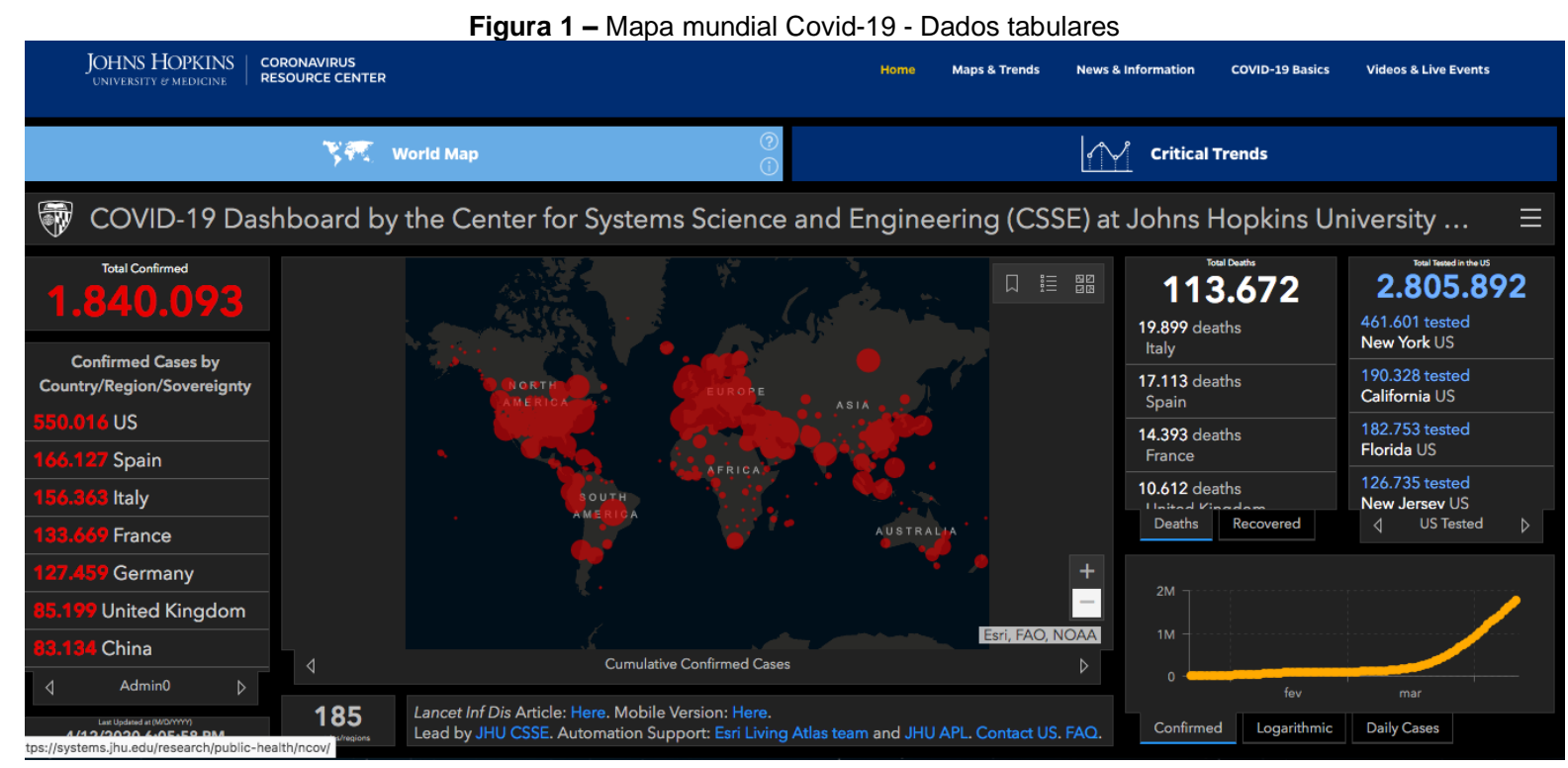

Fonte: Hospital Johns Hopkins (2020).

De acordo com informações disponíveis pelos organizadores do Mapa de Tendências do Coronavírus do Hospital (JOHNS HOPKINS, 2020), este recurso foi elaborado por especialistas em saúde pública para ajudar a melhorar o entendimento do vírus, informar o público e breves formuladores de políticas, a fim de orientar uma resposta, melhorar o atendimento e salvar vidas. A ferramenta serve de apoio à decisão para mitigar pandemias. 
Outra iniciativa interessante com dados tabulares para machine learning é o Kaggle, uma comunidade de aprendizado de máquina pertencente ao Google. O Aprendizado de Máquina (ML) é a programação de computador para que possa 'aprender' as informações disponíveis para eles (SHALEV-SHWARTZ, 2014). Em termos gerais, o aprendizado é o processo de converter dados em experiência ou conhecimento. Nesse processo é realizada a entrada no algoritmo que permite aprendizado de dados de entroncamento, que representa a experiência, e o resultado é uma experiência geral, que geralmente toma a forma do outro programa de computação que pode executar alguma tarefa (SHALEV-SHWARTZ, 2014).

A partir da perspectiva de machine learning, o Kaggle torna possível encontrar códigos e dados necessários para o trabalho da ciência de dados. O Kaggle disponibiliza mais de 19.000 conjuntos de dados públicos e 200.000 cadernos públicos para realizar qualquer análise de dados em pouco tempo.

Figura 2 - Dados tabulares para machine learning do Kaggle

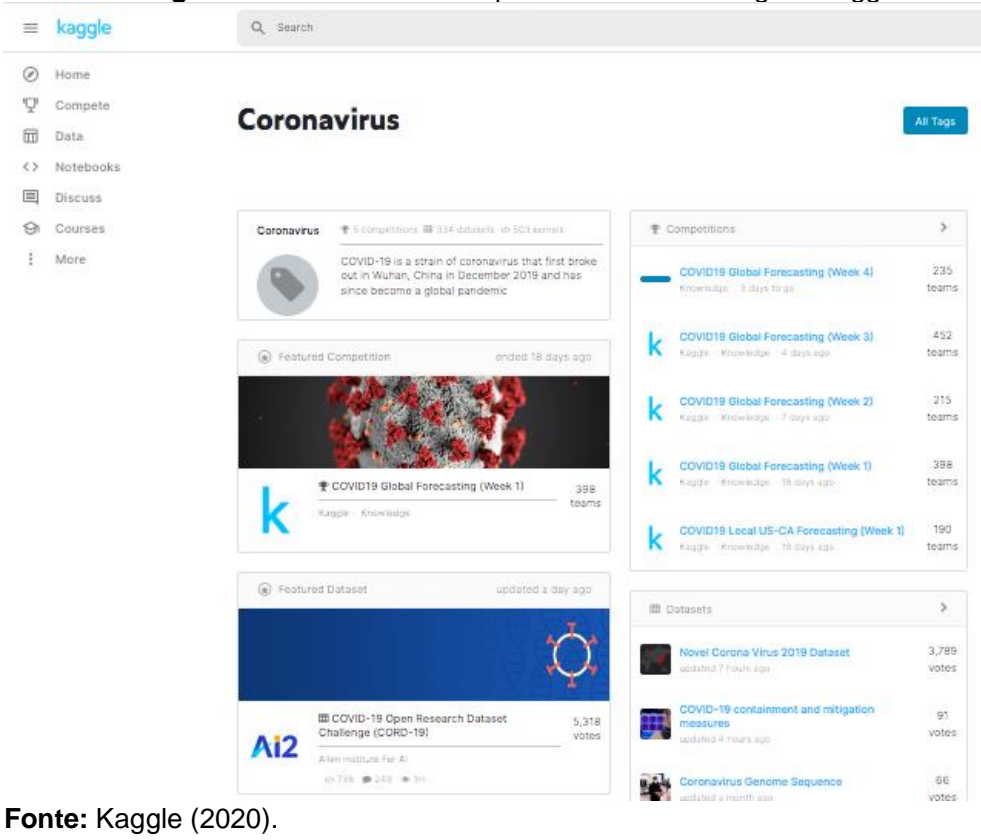

Há um grande corpo de pesquisa e dados em torno da Covid-19. Com isso o Kaglle apresenta várias iniciativas interessantes onde as pessoas podem compor conjuntos de dados para que sejam utilizados em aprendizado de máquina. No contexto da pandemia global da Covid-19, a Kaggle lançou vários desafios para fornecer informações úteis que podem responder a algumas das questões científicas abertas sobre o vírus. É o caso da Previsão Global Covid-19, na qual os participantes são incentivados a ajustar dados mundiais para prever a evolução da pandemia, ajudando a determinar quais fatores afetam o comportamento de transmissão da Covid-19 (KAGGLE, 2020).

Os dados dos artigos de pesquisa aberta da Covid-19 são analisados pela IA do Kaggle e disponibilizados na base Cord 19 e SemanticScholar ${ }^{1}$, um mecanismo gratuito e sem fins lucrativos para pesquisas acadêmicas.

Szafran (2020), do Olhar Digital, publicou que o governo americano informa, por sua vez, que o Cord-19 é a mais extensa coleção de literatura sobre Covid-19 legível por IA em todo o mundo, disponível para mineração de dados e textos. Os pesquisadores podem procurar informações nele e enviar suas respostas pela plataforma, concorrendo a uma recompensa de 1 mil dólares pela melhor resposta.

A ideia por trás em utilizar este tipo de ferramenta é ajudar que pesquisadores tratem dados de maneira mais rápida para fornecer respostar e mitigar a pandemia, considerando a extração de padrões desses dados. Umas das questões em torno da proliferação do vírus é tentar compreender os elementos que potencializam a disseminação, por exemplo. Os estudos têm

${ }^{1}$ Disponível em: https://www.semanticscholar.org/ 
mostrado que fatores como idade e doenças crónicas no paciente o deixam mais vulnerável a Covid-19. Entretanto, o que a inteligência artificial pode ajudar na análise desses dados é identificar se existem outros padrões que podem agravar o quadro de pacientes com o vírus. É justamente esta busca que os algoritmos de aprendizagem de máquinas podem realizar quando trabalhando na análise desses conjuntos de dados tabulares. Daí a importância da veracidade desses dados, como ressaltou o Celso Azevedo em seu blog. É importante que se tenha certeza sobre a procedência desses dados.

\subsection{Processamento de Linguagem Natural}

A Natural Language Processing (NLP) ou processamento de linguagem natural (PLN) se refere, especificamente, a capacidade para que as máquinas possam compreender a linguagem humana e a partir disso possam extrair de textos a informação (significado) contida neles. Esse foi um salto muito importante no aprendizado de máquina e contribuiu para os avanços na computação cognitiva. Segundo Lobo (2018), na medicina existe um terreno fértil para o emprego de sistemas de processamento de linguagem natural visando registrar dados em prontuários eletrônicos, por exemplo.

As técnicas de PLN conferiu a inteligência artificial a oportunidade de utilizar essas ferramentas para extrair informações que estão inseridas em artigos científicos que tratam sobre a Covid-19 e outras pandemias. Algumas dessas iniciativas de processamento de linguagem natural que podem ser destacadas é a CiteNET. É possível pesquisar nessa ferramenta, baseada em PLN, algum texto ou outros documentos relacionados ao coronavírus.

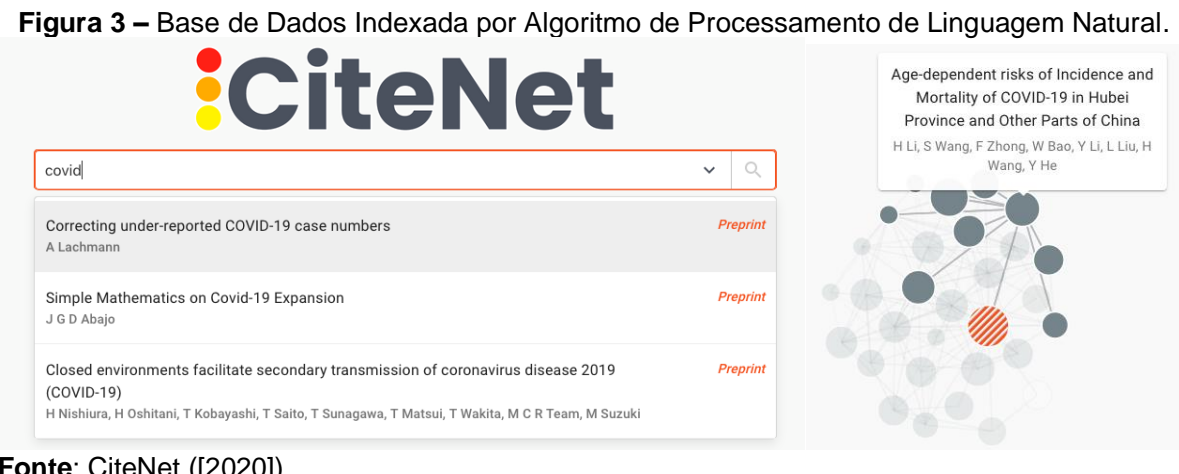

Segundo nota do site da CiteNet essa ferramenta está, atualmente, em desenvolvimento. No entanto, devido à pandemia de Covid-19, a CiteNet optou por disponibilizar a ferramenta ao público e indexar o bioRxiv e o medRxiv diariamente. A intenção é fornecer uma ferramenta útil e atualizada de pesquisa de literatura para a comunidade científica.

Do ponto de vista de áreas como a Ciência da Informação, iniciativas como esta são extremamente importantes porque a todo o momento muitos artigos são publicados e, dependendo da sua estratégia de busca para recuperação da informação, a identificação de produções de alto impacto pode ser morosa. No contexto atual, toda a comunidade científica está trabalhando incessantemente para descobrir padrões de disseminação viral e entendimento de como o vírus se comporta nos diferentes países, por exemplo. Desse modo, é praticamente impossível para os médicos se manterem atualizados com todo o material que está sendo publicado.

\subsection{Visão computacional}

$\mathrm{Na}$ área da Saúde, a visão computacional tem encontrado terreno fértil, no campo da imagem médica digital. A imagem médica digital é uma função $f(x, y)$ em escala de níveis de cinza particionada em coordenadas espaciais que pode ser representada por uma matriz, cujos índices de linhas e de colunas identificam um ponto de imagem (pixel) (SANTOS; FERREIRA JUNIOR; WADA; TENÓRIO; BARBOSA; MARQUES, 2019, p.696). No contexto da visão computacional, encontra-se a capacidade das máquinas extraírem dados e informações a partir de imagens.

A visão computacional no combate à Covid-19, possui ações centradas em análises de exames e diagnóstico processados por imagem. Como exemplo, alguns bancos de dados de imagens. O GitHub disponibiliza um banco de imagens para 
treinamentos, envolvendo técnicas de processamento de imagem, visão computacional e aprendizado de máquina. Disponibiliza também um repositório de dados sobre o novo coronavírus e a Covid-19 (2019-nCoV) com dados visuais que são alimentados pelos laboratórios do Hospital Johns Hopkins.

Figura 4 - Banco de imagens para treinamento com cases do coronavírus.

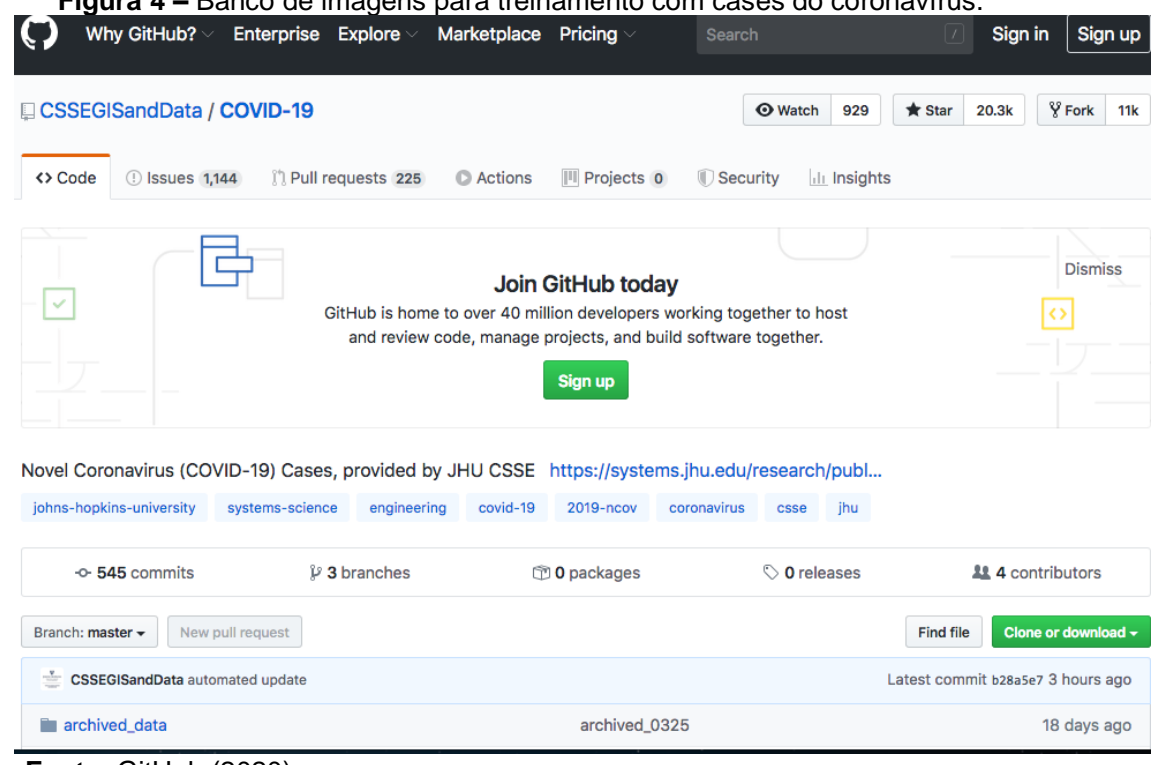

Fonte: GitHub (2020)

Pontuamos também a base de imagens desenvolvida pela Sociedade Italiana de Radiologia Médica e Intervencionista, que realiza análise utilizando visão computacional (SOCIETÀ ITALIANA DI RADIOLOGIA MEDICA E INTERVENTISTICA, 2019). A base possui foco especial no uso do big data e da inteligência artificial nas ciências da vida, no desenvolvimento da medicina de precisão e na digitalização e otimização dos processos, principalmente, com ênfase no banco de imagens dos hospitais filiados. O "big data" gradualmente vem sendo introduzido no sistema de atenção à saúde (LOBO, 2018). O volume de dados "[...] em saúde vem crescendo em um ritmo acelerado nos últimos anos, caracterizando o que alguns autores chamam de [...] era do big data" da saúde" (SANTOS; FERREIRA JUNIOR; WADA; TENÓRIO; BARBOSA; MARQUES, 2019, p. 394).

Figura 5 - Base de imagem computacional

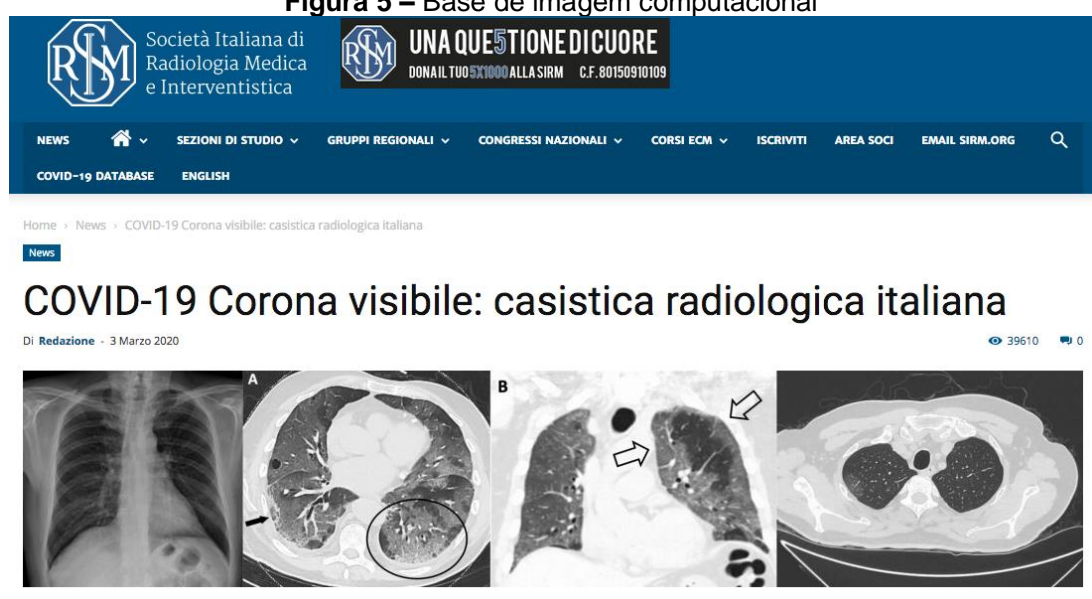

Fonte: Società Italiana di Radiologia Medica e Interventistica (2020)

O Data Life (2020) é uma iniciativa brasileira que é uma das divisões da Data $H$. Trabalha em parceria com algumas entidades da área médica, a partir do uso de um algoritmo de detecção de pneumonia em imagens de pulmões. O objetivo é a criação de um dataset que permita realizar a identificação de enfermidades em tomografias de pulmão. 


\section{Principais aplicações da Inteligência Artificial no combate ao novo coronavírus}

Alguns especialistas (LOBO, 2018; SANTOS; FERREIRA JUNIOR; WADA; TENÓRIO; BARBOSA; MARQUES, 2019; ZHEN, 2019) têm apontado a inteligência artificial com impacto tão significativo na sociedade como o que o mundo viu com a energia elétrica. E o primeiro vislumbre prático do seu alcance está sendo possível perceber neste momento de expansão da Covid19. Estamos diante da primeira Pandemia onde a inteligência artificial está tendo participação expressiva enquanto aplicação.

No momento do SARS não se tinha a IA no estágio que ela se encontra hoje. A inteligência artificial está permitindo os cientistas apresentarem resultados rápidos e interessantes. Se observarmos a velocidade de resposta das empresas de tecnologia e saúde à ameaça da Covid-19, é possível perceber que tem sido mais rápida do que em epidemias anteriores.

A IA tem permitido averiguar todos os aspectos que permitem indicar a evolução de uma epidemia para uma pandemia. Diante de um desafio como este, a primeira grande questão a ser respondida é como identificar que uma epidemia está se transformando em uma pandemia. Com a inteligência artificial foram dados os primeiros sinais de alerta por revistas especializadas, a exemplo da Wired.

Desse modo, a primeira forma de aplicação da IA teve o uso de comparações que foram feitas por uma startup Canadense, a Blue Dots, cruzando dados do novo coronavírus na cidade de Wuhan-China com o que havia acontecido com o SARS em 2003. Muitos destes dados foram vasculhados na mídia e nos tikets de viagem que apontavam o transito de saída de doentes da cidade epicentro. O cruzamento desses dados com os voos que saiam de Wuhan no período, permitiu que fossem indicadas as próximas localidades que teriam disseminação dessa pandemia. As comparações apontaram para o potencial de um risco grande para pandemia.

A Blue Dots foi criada em 2013 por Kamran Khan, um médico infectologista que trabalhou em hospitais no Canadá durante o surto da SARS. A startup que tem 40 funcionários previu corretamente que a doença iria chegar a Bangkok, Seul, Taipei e Tóquio. Com o apoio da IA teve êxito e, apesar de o governo chinês não ter fornecido muitas informações para as autoridades globais de saúde, a exemplo da Organização Mundial de Saúde (OMS), foi possível prever a expansão do coronavírus com base em outro fator de risco: a emissão de passagens aéreas (AGRELA, 2020). Desse modo, nove dias antes da OMS emitir o alerta de epidemia, esta startup de IA detectou a doença e já sabia para quais países a doença iria viajar. 
Figura 6 - Resposta da Covid-19 comparada com outras epidemias de 2003 a 2019

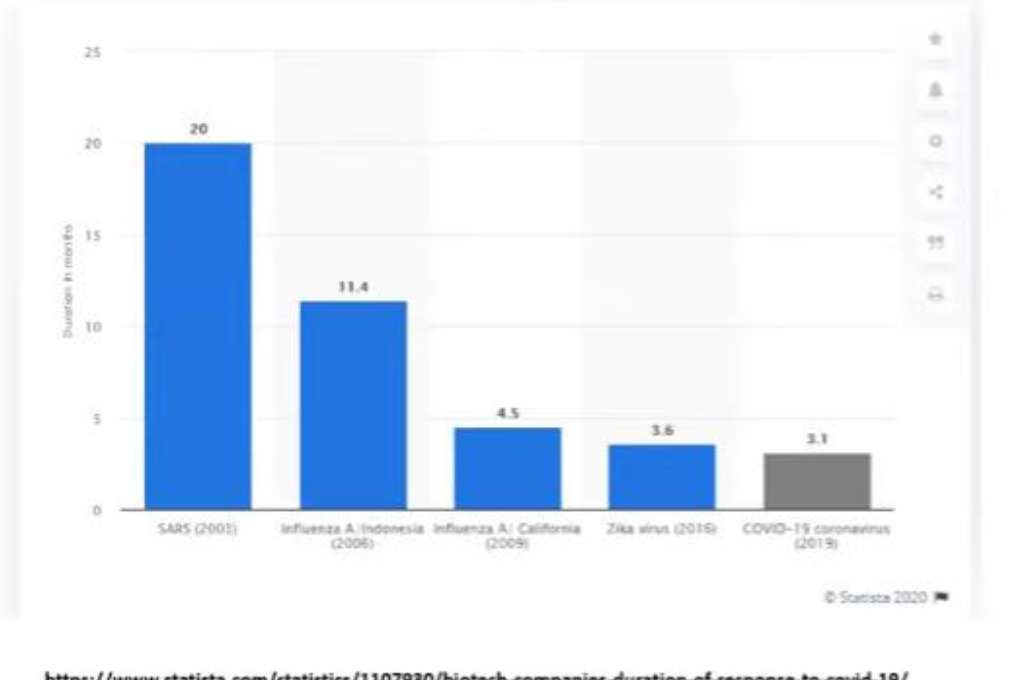

Fonte: Statista (2020).

A segunda forma de aplicação da IA no combate ao vírus permitiu perceber como a pandemia se dissemina entre os países. Esse rastreamento é feito pelo algoritmo olhando para o passado. Uma aplicação da Nexframe observou a possibilidade de mutação da Covid-19 que é realizada a cada dois meses. Essa identificação é feita a partir da assinatura do DNA da doença. Essa análise busca descobrir onde surgiu a mutação inicial do vírus. E assim, a IA é capaz de determinar onde a contaminação está sendo feita por transmissão local ou por viagem.

Figura 7 - Uso de inteligência artificial para identificar a mutação da Covid-19
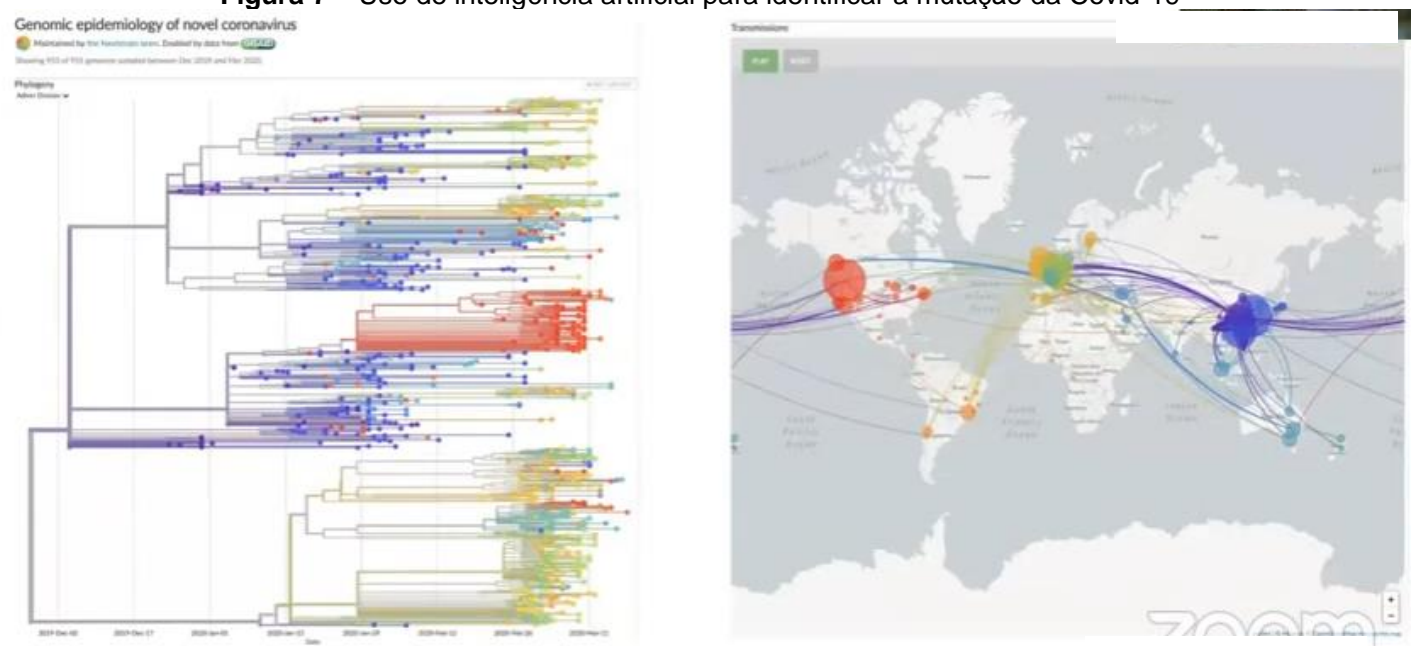

Nota: as cores representam as mutações do genoma do vírus.

Fonte: i2ia (2020)

Uma terceira aplicação da IA realiza a analise de documentos frutos de pesquisa sobre a família do vírus. Especificamente, na primeira quinzena de abril de 2020, sobre a Covid-19, já se tinha um pouco mais que 6.000 documentos que precisavam ser analisados e sincronizados. Aqui entrou a aplicação da "Data H" com o uso de linguagem natural na mineração, sincronismo e correlação de milhares de documentos, facilitando a identificação de avanços e estruturação de novos protocolos.

Considerando o avanço exponencial que a pandemia está tendo nos países infectados e a produção das pesquisas que ocorrem simultaneamente, a quarta forma de aplicação da inteligência artificial busca facilitar o processo de diagnóstico. 
Existem vários tipos de testes de diagnóstico, a exemplo da reação imunológica e os mais sofisticados que passam por exames de imagem. A análise de imagem é um dos campos que mais se desenvolve na Medicina com o uso de tecnologias de deep learning ou aprendizado profundo. De acordo com Santos; Ferreira Junior; Wada; Tenório; Barbosa; Marques (2019), aplicações de aprendizado profundo é uma das áreas de inteligência artificial mais recentes que vem ganhando atenção da comunidade científica e sendo, paulatinamente, incorporada no processamento de imagens.

Um algoritmo desenvolvido na China prescreve decisões a partir de análises de imagens de pulmões, visando identificar aqueles que sofreram de pneumonia causada por coronavírus, conforme ilustração na Figura 8.

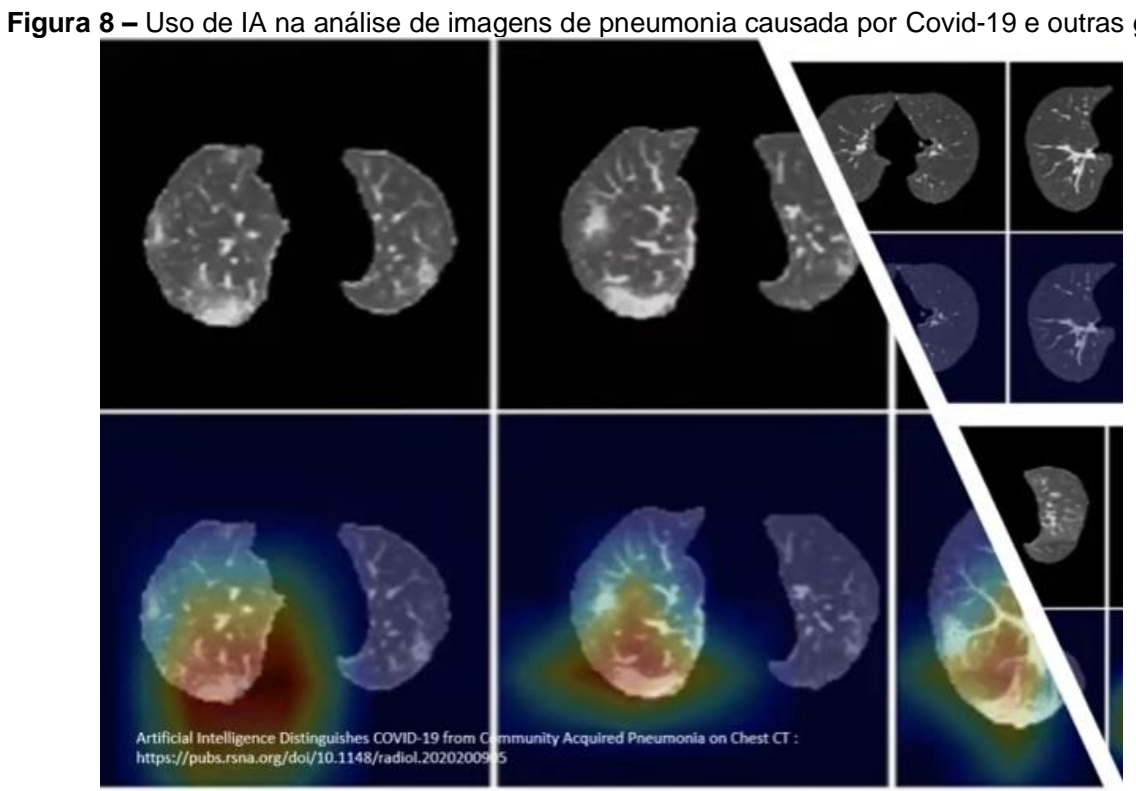

Fonte: Santos; Ferreira Junior; Wada; Tenório; Barbosa; Marques (2019).

Pesquisadores da Universidade de Nova lorque em parceria com médicos chineses desenvolveram um algoritmo de IA que permite identificar e cruzar outros fatores do paciente - como idade, nível de hemoglobina, enzima no fígado, etc. - que interferem ou sofrem variações no sujeito infectado. Esses elementos são tratados pelo algoritmo procurando identificar nos pacientes esses e outros indicadores de perigo que podem levar a potencial de contagion e a fatalidade (ZHEN, 2019). De acordo com Zhen (2019), os modelos que eles construíram demonstraram ter uma precisão de 70 a $80 \%$ na previsão de casos graves.

Zhen (2019) em seu artigo publicado em três de abril 2019 e intitulado Al could help identify coronavirus patients at greatest risk of becoming seriously ill, visa mostrar qual dos pacientes identificados com a Covid-19 tem maiores chances de desenvolver crises severas respiratórias e ficar gravemente doente. Dentre outros aspectos que uma aplicação como esta pode contribuir, destaca-se a possibilidade de planejamento para equipagem de leitos e viabilização de hospitais de campanha, além de outros direcionamentos como alocação de gastos públicos.

A sexta aplicação da IA no combate a Covid-19 está voltada à elaboração de uma vacina contra o vírus. Os pesquisadores do MIT usaram uma modelagem de deep learning para identificar um medicamento chamado halicina que mata muitas bactérias. De acordo com Trafton (2020), a halicina (linha superior da figura a seguir) impediu o desenvolvimento de resistência a antibióticos em coronavírus, enquanto a ciprofloxacina (linha inferior) não. 
Figura 9 - Pesquisadores do MIT usa aprendizado de máquina para emular uma vacina para a Covid-19

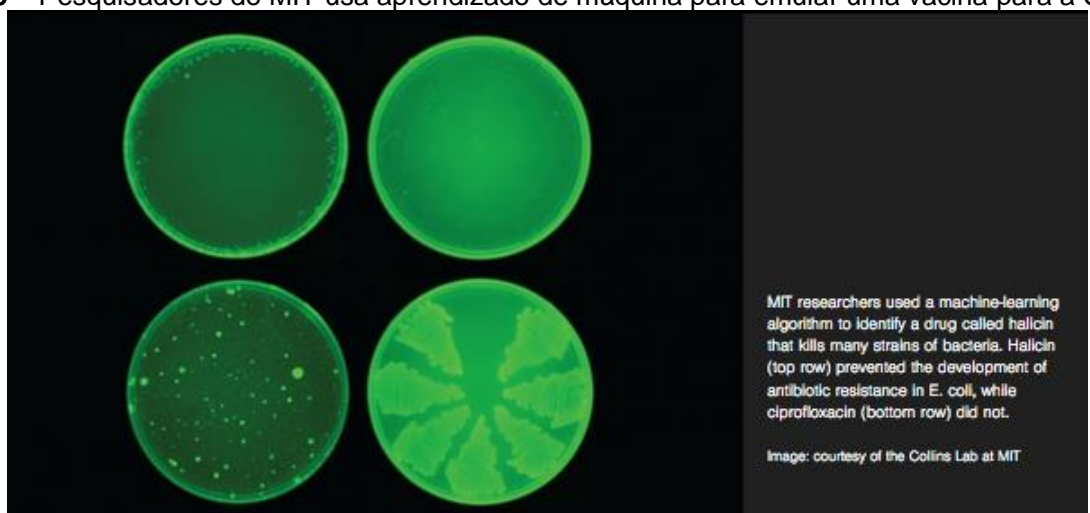

Fonte: Trafton (2020).

"Queríamos desenvolver uma plataforma que nos permitisse aproveitar o poder da inteligência artificial para inaugurar uma nova era de descoberta de antibióticos", disse o responsável pelo estudo, James Collins, professor de ciência e engenharia médica do Termeer no Instituto de Engenharia e Ciência Médica do MIT (IMES) e do Departamento de Engenharia Biológica (TRAFTON, 2020, documento em linha).

A Revista Nature também apresentou um estudo no sentido da produção de novas drogas eficazes no combate ao vírus, com foco de exploração pela indústria farmacêutica. $O$ aprendizado de máquina e o big data estão ajudando os químicos a pesquisar no vasto universo químico por melhores medicamentos. 
Figura 10 - Resultado do Chemical Cosmo - Jogo entre químicos e um algoritmo para descobrir novas drogas farmacêuticas

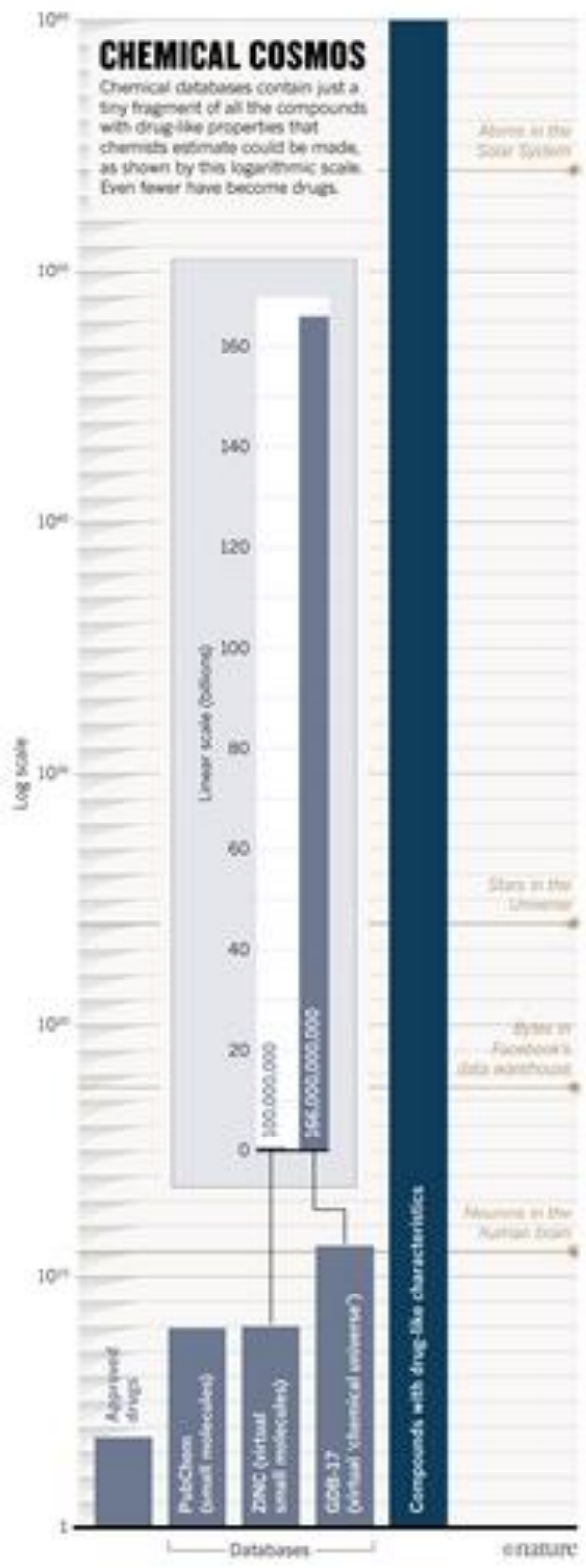

Fonte: Mullard (2017).

Em 2016, a empresa farmacêutica Sunovion atribuiu a um grupo de funcionários experientes uma tarefa incomum, um jogo para descobrir novos medicamentos.

Na sede da empresa em Marlborough, Massachusetts, todos os químicos foram convidados a jogar um jogo para ver quem poderia descobrir as melhores pistas para novos medicamentos. Em suas estações de trabalho, havia uma grade de centenas de estruturas químicas, das quais apenas dez eram rotuladas com informações sobre seus efeitos biológicos. Os especialistas tiveram que selecionar outras moléculas que poderiam vir a ser candidatas a medicamentos, usando seu conhecimento suado de estrutura química e biologia. Dos 11 jogadores, 10 lutaram na tarefa por várias horas. Mas um passou rapidamente em milissegundos - porque era um algoritmo (MULLARD, 2017, p. 1).

Os químicos que concorreram com o algoritmo levaram mais de 10 horas para solucionar os compostos moleculares, enquanto que $o$ algoritmo levou alguns segundos. 
Outros exemplos de IA que estão sendo utilizados pelo mundo na área da saúde, vale salientar o robô dinamarquês, do tipo veiculo autônomo, que realiza a descontaminação dos ambientes. Ele joga raios de luz ultravioleta que tem a função de quebrar moléculas. Uso de computação cognitiva na gestão hospitalar para ajudar na eficiência. Questões como troca de turnos, avisos entre funcionários, uso de medicação equivocadas, dentre outros. Utilização de assistentes virtuais para pacientes enfermos, visando diminuir os efeitos da solidão causadas durante os períodos de quarentena, por exemplo.

Como é possível perceber, a inteligência artificial, em seus diferentes tipos, tem atuado nas etapas da curva da Covid-19, desde o contágio, passando pelo ápice até a elaboração da vacina. Contudo, especialistas chamam atenção sobre a necessidade de se pensar o uso da inteligência artificial também nos efeitos causados na sociedade pela pandemia.

Desse modo, é possível verificar mudanças comportamentais significativas, desde à condução política por parte de alguns países, até a condução de práticas de saúde coletiva, aspectos com relação ao impacto econômico e até posicionamentos nas redes sociais. É possível perceber que a inteligência artificial pode atuar de forma intensa em:
1) Telemedicina
2) Home work
3) Melhor eficácia de drogas (medicina personalizada)
4) Identificação mais rápida de doenças raras por meio de fotos faciais
5) Identifica a doença antes que ela aconteça por meio de padrões metabólicos do sujeito, exemplo do Alzheimer.

O que está acontecendo no momento com o paradigma do coronavirus demonstra que o mundo apresentará mudanças significativas, ressaltando o que o Biólogo Atila lamarino, no Programa Roda Viva de 30 de março de 2020, "após a Covid-19 teremos uma nova forma de nos relacionar com o mundo". Segundo Cezar Taurion (2020), essas transformações serão ainda mais drásticas nos campos da saúde, do ponto de vista da inovação e da mudança de comportamentos motivados, principalmente, pela inteligência artificial. A IA no cuidado com a saúde vem gerando transformações significativas no que tange:

a) auxílio de tarefas monótonas;

b) design de tratamento;

c) chat boots;

d) mineração de registros médicos;

e) assistentes de cuidados visual.

Essas tecnologias inauguram um novo momento na sociedade e no repensar o trato, a informação e a inovação em saúde. E aos poucos, os cidadãos vão percebendo a transformação digital que estamos presenciando e, principalmente, reconhecendo os alcances, potenciais e implementações da inteligência artificial nesse contexto (TAURION, 2018).

No Brasil, o Ministério da Saúde sinaliza intensão em usar Big Data para cruzar dados. Em pronunciamento, anunciou que irão aplicar big data nas informações das unidades de saúde, que estão sendo conectadas. A Secretaria de Estado de Saúde do Amazonas também anunciou a criação de um 'BIG Data da Saúde', para integrar as informações produzidas em hospitais, prontos-socorros e demais unidades da capital e interior e ajudar no planejamento de ações e nas tomadas de decisão da nova gestão. Dentre as ações brasileiras, destaca-se também que o Hospital Albert Einstein soltou um desafio no Kaggle para identificar os pacientes que possuem maior chance de desenvolver um quadro mais grave.

De acordo com Neves (2020) é possível ainda destacar no Brasil, uma iniciativa de curadoria digital da ACIB, que está desenvolvendo seleção de conteúdo específico sobre o coronavírus a partir de notícias, informações, orientações e serviços divulgados com intuito de superar a crise. 


\section{Considerações Finais}

Até o momento a sociedade apresenta dificuldades para entender os benefícios da IA. Um estudo como este não poderia chegar a mais certezas que questionamentos. Desse modo, este artigo termina suscitando novas perguntas, como: $O$ uso da IA na saúde e política pública pode mudar a visão que a sociedade tem refletido? A sociedade percebe a IA como aliada? As populações sabem quando estão utilizando a IA? Como os médicos encaram a IA? As pessoas entendem que a IA não é uma entidade?

Nesse momento a telemedicina e a e-learning ganharam visibilidade e se expandem a passos largos. Em se tratando da IA na área de Saúde, observamos que no leque das tendências devem se destacar, nos próximos anos, os campos do processamento da linguagem natural e dos assistentes pessoais, tornando possíveis novas formas de interação entre médico e paciente e acompanhamento de tratamentos dentro e fora de instituições médicas. Também o uso da realidade virtual e realidade aumentada podem ser promissoras quando associadas a telemedicina, por exemplo. A inteligência artificial termina por permear de forma transversal esses movimentos.

Na perspectiva acadêmica, os pesquisadores de vanguarda da IA direcionam seus esforços para a chamada General Al. Uma inteligência hipotética de uma máquina que potencializa a capacidade do aprendizado de qualquer tarefa intelectual que um ser humano possa realizar. Uma inteligência artificial mais genérica que não somente execute tarefas separadas, mas sim emule a inteligência humana para realizar uma linha de aprendizado mais complexo.

Existe um movimento cíclico entre as tecnologias, a inovação e o comportamento. Essas forças se retroalimentam e não estão isoladas. Assim, os novos usos estão ligados aos movimentos que a sociedade irá realizar a partir dos seus novos hábitos frente ao cenário que se desenha no mundo com a expansão dessa pandemia e a transformação digital que está em curso. Essas evoluções vão acontecer oriundas das necessidades da sociedade. Uma coisa é certa, parafraseando o Renato Russo: "O futuro não é mais como era antigamente".

\section{Referências}

AGRELA, Lucas. Inteligência artificial previu epidemia do coronavírus da China: startup canadense previu a quais países a doença chegaria. Exame, 28 jan. 2020. Disponível em: https://exame.abril.com.br/tecnologia/inteligencia-artificial-previu-epidemia-do-coronavirusda-china/. Acesso em: 18 abr. 2020.

CITENET. [2020]. Disponível: https://citenet.io/. Acesso em: 12 jul. 2020.

DATALIFE. Ribeirão Preto, 2020. Disponível em: https://pt.datalife.ai/portugueshome. Acesso em: 12 jul. 2020.

FERNANDES, Fernando Timóteo; CHIAVEGATTO FILHO, Alexandre Dias Porto. Perspectivas do uso de mineração de dados e aprendizado de máquina em saúde e segurança no trabalho. Revista Brasileira de Saúde Ocupacional, São Paulo, v. 44,12 p., 2019. DOI: https://doi.org/10.1590/2317-6369000019418. Disponível em: https://www.scielo.br/scielo.php?script=sci arttext\&pid=S030376572019000101401. Acesso em: 24 jun. 2020.

GITHUB. CSSEGISandData / COVID-19. 2020. Disponível: https://github.com/CSSEGISandData/COVID-19 Acesso em: 12 jul. 2020.

HEURYS. 2020. Disponível em: http://www.heurys.com.br/. Acesso em: 12 jul. 2020.

INTERNATIONAL Association of Artificial Intelligence. i2ia. Guia rápido de inteligência artificial, 2020. Disponível: https://conteudo.i2ai.org/guiadeia. Acesso em: 24 jun. 2020.

HOSPITAL JOHNS HOPKINS. Word Map. Covid-19 Dashboard by the Center for Systems Science and Engineering (CSSE) at Johns Hopkins University (JHU). 2020. Disponível em: https://coronavirus.jhu.edu/map.html. Acesso em: 12 abr.2020.

KAGGLE. Coronavirus. 2020. Disponível em: https://www.kaggle.com/tags/covid19. Acesso em: 12 abr.2020.

LAVILLE, Christian; DIONE, Jean. A construção do saber: manual de metodologia da pesquisa em ciências humanas. Porto Alegre. Editora Artes Médicas; Belo Horizonte: EditoraUFMG, 1999.

LEAL NETO, Onício Batista; ALBUQUERQUE, Jones; SOUZA, Wayner Vieira; CESSE, Eduarda; CRUZ, Oswaldo Gonçalves. Inovações disruptivas e as transformações da saúde pública na era digital. Cadernos de Saúde Pública, Rio de Janeiro, v. 33, n. 1, 4 p., 2017. DOI: https://doi.org/10.1590/0102-311x00005717. Disponível em: https://www.scielo.br/scielo.php?pid=S0102311X2017001100501\&script=sci arttext. Acesso em: 12 abr. 2020.

LIMA, Clóvis Ricardo Montenegro de; FINAMOR, Márcio da Silva. Bibliotecários em hospitais: o olhar crítico e humanístico da profissão. In: LIMA, Clóvis Ricardo Montenegro de. Saúde e discurso. Rio de Janeiro: Salute, 2019. p. 118-135. Disponível em:

https://ridi.ibict.br/bitstream/123456789/1019/7/Saude.pdf. Acesso em: 24 jun. 2020. 
LOBO, Luiz Carlos. Inteligência artificial, o futuro da Medicina e a Educação Médica. Revista Brasileira de Educação Médica, Brasília, v. 42, n. 3, p. 3-8, set. 2018. DOI: https://doi.org/10.1590/1981-52712015v42n3rb20180115editorial1. Disponível em: http://www.scielo.br/scielo.php?script=sci arttext\&pid=S0100-55022018000300003\&lng=en\&nrm=iso. Acesso em: 19 abr. 2020.

MULLARD, Asher. O guia do farmacêutico para a galáxia. Nature, 26, set. 2017. Disponível em: https://www.nature.com/articles/s41570019-0124-0?draft=collection. Acesso em: 12 abr. 2020.

NEVES, Bárbara Coelho. Tecnologias de inteligência artificial utilizadas na saúde durante a pandemia de Covid-19. Observatório da Imprensa: Coronavírus, ano 20, n. 1091, ed. 1086, 5 maio 2020. Disponível em:

http://www.observatoriodaimprensa.com.br/coronavirus/tecnologias-de-inteligencia-artificial-utilizadas-na-saude-durante-a-pandemia-decovid-19/. Acesso em: 23 jun. 2020.

SANTOS, Marcel Koenigkam; FERREIRA JUNIOR, José Raniery; WADA, Danilo Tadao; TENÓRIO, Ariane Priscilla Magalhães; BARBOSA, Marcello Henrique Nogueira; MARQUES, Paulo Mazzoncini de Azevedo. Inteligência artificial, aprendizado de máquina, diagnóstico auxiliado por computador e radiômica: avanços da imagem rumo à medicina de precisão. Radiologia Brasileira, v. 52, n. 6, p. 387-396, nov./dez. 2019. DOI: https://doi.org/10.1590/0100-3984.2019.0049. Disponível em: http://www.scielo.br/pdf/rb/v52n6/pt 0100-3984-rb20190049.pdf. Acesso em: 18 abr. 2020.

SHALEV-SHWARTZ, Shai. Understanding Machine Learning. Cambridge: University Press, 2014.

SOCIETÀ ITALIANA DI RADIOLOGIA MEDICA E INTERVENTISTICA. Digitalizzazione e Intelligenza Artificiale. Le applicazioni nel settore delle Life-Sciences. Itália, 9 luglio 2019. Disponível em: https://www.sirm.org/2019/07/09/digitalizzazione-e-intelligenza-artificiale-leapplicazioni-nel-settore-delle-life-sciences/. Acesso em: 12 jul. 2020.

SOCIETÀ ITALIANA DI RADIOLOGIA MEDICA E INTERVENTISTICA. Covid-19: casistica radiologica italiana. Itália, 2020. Disponível em: https://www.sirm.org/category/senza-categoria/covid-19/ Acesso em: 12 jul. 2020.

STATISTA. 2020. Disponível em: https://www.statista.com/. Acesso em: 12 jul. 2020.

SZAFRAN, Vinícius. Inteligência artificial vai analisar 29 mil pesquisas sobre Covid-19 para encontrar respostas. Olhar Digital, 2020. Disponível em: https://olhardigital.com.br/coronavirus/noticia/inteligencia-artificial-vai-analisar-29-mil-pesquisas-sobre-covid-19-paraencontrar-respostas/98220. Acesso em: 12 abr. 2020.

TAURION, Cezar. A Inteligência Artificial está perto do seu ponto de inflexão. ClO from IDG, 2018. Disponível em: https://cio.com.br/ainteligencia-artificial-esta-perto-do-seu-ponto-de-inflexao/. Acesso em: 24 jun. 2020.

TRAFTON, Anne. Artificial intelligence yields new antibiotic. MIT News Office, Feb. 20, 2020. Disponível em: http://news.mit.edu/2020/artificial-intelligence-identifies-new-antibiotic-0220. Acesso em: 11 abr. 2020.

ZHEN, Liu. Al could help identify coronavirus patients at greatest risk of becoming seriously ill. South China Morning Post. Beijing, 3 abr. 2019. Disponível em: https://www.scmp.com/news/china/science/article/3078378/ai-could-help-identify-coronavirus-patients-greatest-risk. Acesso em: 10 abr. 2019. 